\title{
ANÁLISE COMPARATIVA DE FUSÃO DE IMAGENS DOS SENSORES ORBITAIS CCD E HRC UTILIZANDO IHS E COMPONENTES PRINCIPAIS NA REGIÃO URBANA DO MUNICÍPIO DE TRÊS LAGOAS MS/BR
}

\author{
Cesar Cardoso Ferreira ${ }^{1}$
}

Edson Luis Piroli ${ }^{2}$

Arnaldo Yoso Sakamoto ${ }^{3}$

\begin{abstract}
RESUMO
Este trabalho tem como objetivo analisar dados obtidos por meio da transformação de Intensity Hue Saturation (IHS) e Componentes Principais para combinação de imagens de diferentes sensores orbitais e resolução espacial na área urbana do município de Três Lagoas, localizado na região leste do estado de Mato Grosso do Sul. Foram utilizados imagens do satélite CEBRS 2B (China Brazil Earth Resource Satellite) dos seus sensores Charge Coupled Device (CCD) e High Resolution Camera (HRC) processadas em ambiente de Sistema de Informação Geográfica. Observou-se como resultado que a técnica da IHS apresentou maior nitidez em relação a técnica Componentes Principais, mas ambas as imagens fusionadas apresentaram um melhor aproveitamento das informações geradas pelos diferentes sensores. Conclui-se que, as técnicas de fusão analisadas contribuíram para uma melhor definição dos alvos observados nas imagens, colaborando para a sua interpretação.
\end{abstract}

PALAVRAS-CHAVE: Sensoriamento remoto, fusão de imagens orbitais.

\section{ANALYSIS IMAGE SENSOR FUSION COMPARATIVE ORBITAL CCD and HRC IHS USING AND MAIN COMPONENTS IN THE REGION OF THREE URBAN COUNTY LAKES MS / BR}

\begin{abstract}
This work aims to analyze data obtained through the transformation of Intensity Hue Saturation (IHS) and Principal Component for combining images from different satellite sensors and spatial resolution in the urban area of the municipality of Três Lagoas, located in eastern Mato Grosso do Sul state. Satellite images were used CEBRS 2B (China Brazil Earth Resource Satellite) sensors Charge Coupled Device (CCD) and High Resolution Camera (HRC) processed in a Geographical Information System environment. It was observed that as a result the technique of IHS presented more clearly in relation to technical principal components, but both images fused showed a better use of information generated by the different sensors. In conclusion, the fusion techniques analyzed contributed to a better definition of targets observed in the images, adding to its interpretation.

KEYWORDS: Remote sensing, orbital images fusion.

\footnotetext{
${ }^{1}$ Doutorando em Geografia pela UNESP-Presidente Prudente e Técnico em Cartografia pela UFMS-Três Lagoas

${ }^{2}$ Professor Doutor pela UNESP de Ourinhos

${ }^{3}$ Professor Doutor pela UFMS de Três Lagoas
} 


\section{INTRODUÇÃO}

O uso dos dados orbitais é crescente no âmbito da análise geográfica e suas atribuições, resultados e produtos tem sido de fundamental importância para a pesquisa científica e trabalhos técnicos. No caso desta pesquisa os dados orbitais vêm nos atender com informações obtidas por meio de sensores que obtém imagens da superfície terrestre de diferentes resoluções espaciais.

Os sensores para observação terrestre se tornam cada vez mais utilizado em função da facilidade de aquisição e processamento destas informações, "Utilização de sensores para a aquisição de informações sobre objetos ou fenômenos sem que haja contato direto entre eles" (NOVO, 2008).

No intuito de desenvolver tecnologia em sensores, Brasil e China assinaram um acordo em 6 de julho de 1988, para a construção de um satélite de recursos terrestres denominado de CBERS (China-Brazil Earth Resources Satellite), hoje o programa já está em seu terceiro satélite denominado CBERS 2B, o qual é um dos poucos satélites que seu produto é disponibilizado gratuitamente aos seus usuários, através do site do INPE (Instituto de Pesquisas Espaciais).

Para melhor visualização dos dados gerados por diferentes sensores, alguns procedimentos de processamento de imagens têm sido desenvolvidos e aplicados. Alguns destes procedimentos combinam imagens de diferentes características espectrais e espaciais para sintetizar uma nova imagem com melhor resolução espacial do que a imagem multiespectral original. A estas operações dá-se o nome de fusão de imagens.

Segundo Wald (1999), a fusão de dados orbitais pode ser caracterizada como uma estrutura de trabalho em geoprocessamento onde ferramentas e técnicas são desenvolvidas com o objetivo de combinar informações advindas de diferentes sensores, onde a utilização dessa técnica permite a obtenção de uma imagem de melhor qualidade.

Desta forma, foram desenvolvidas técnicas de processamento de imagens, tais como: IHS (Intensity, Hue, Saturation) e Componentes Principais, utilizadas no presente trabalho.

O IHS pode ser comparado com o olho humano que enxerga imagens sem 
distinguir o vermelho, azul e verde (RGB), mas sim a intensidade, o matiz e a saturação das cores (SPRING, 2006).

Segundo a Divisão de Processamento de Imagens (DPI) do INPE (2015) a intensidade ou brilho é a medida de energia total envolvida nos comprimentos de onda, sendo responsável pela sensação de brilho da energia incidente sobre o olho. A matiz ou cor de um objeto é a medida do comprimento de onde médio da luz que se reflete ou se emite, definindo, a cor do objeto. Já a saturação ou pureza, expressa o intervalo de comprimento de onda ao redor do comprimento de onda médio, no qual a energia é refletida ou transmitida. Um alto valor de saturação resulta em uma cor espectralmente pura, ao passo que um baixo valor indica uma mistura de comprimentos de onda produzindo tons pastéis (apagados).

Componentes Principais é uma técnica de realce que reduz ou remove esta redundância espectral, ou seja, gera um novo conjunto de imagens cujas bandas individuais apresentam informações não-disponíveis em outras bandas (SPRING, 2006).

De acordo com Maikon (2008), as imagens de sensoriamento remoto tem ampliado suas aplicações nos últimos anos devido à sua melhor qualidade e maior disponibilidade, permitindo observar a superfície terrestre com maior detalhe e freqüência. A integração destas imagens com outras fontes de dados espaciais em Sistemas de Informação Geográfica (SIG) aumentaram significativamente as opções e possibilidades em análises espaciais de dados da superfície terrestre.

\section{OBJETIVOS}

Aplicar e analisar a técnica de fusão de imagens dos sensores orbitais CCD e HRD utilizando os procedimentos IHS e Componentes Principais.

\section{ÁREA DE ESTUDO}

O recorte espacial compreende-se um quadrante da área urbana do município de Três Lagoas/MS entre os paralelos 2043'29"S, $20^{\circ} 50^{\prime} 35^{\prime \prime} \mathrm{S}$ e os

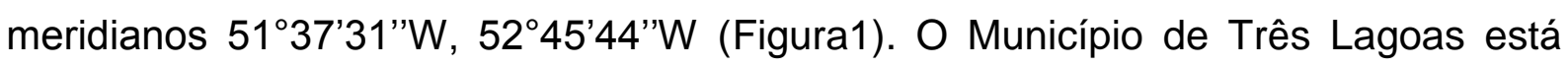
localizado na região leste do estado de Mato Grosso do Sul na divisa com o estado de São Paulo. 
Figura 1: Localização da área de estudo

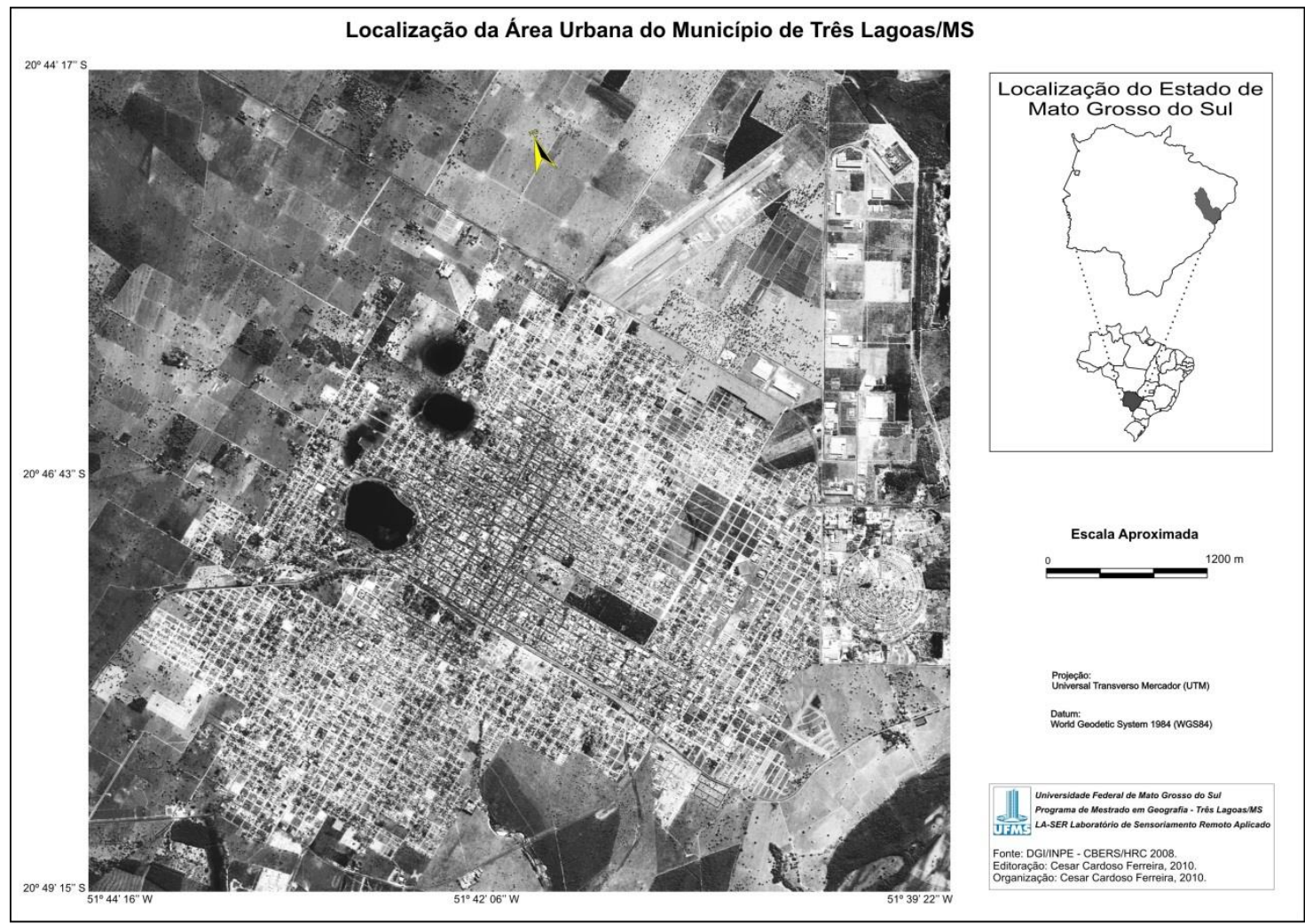

Fonte: CBERS HRC, 2008. Organização: Cesar Cardoso Ferreira

\section{MATERIAIS E PROCEDIMENTOS OPERACIONAIS}

Para a realização da pesquisa foram utilizados imagens do satélite CBERS 2B sensor CCD bandas 2, 3 e 4, do ano de 2008 órbita 160 e ponto 123 e imagens do sensor HRC banda pancromático do ano de 2008 órbita 160c e ponto 1234 (Quadro 1) além de GPS, Hardware e Software Spring. Os critérios utilizados para selecionar as imagens foram: disponibilidade, percentual de nuvens e proximidade das datas nas imagens orbitais dos sensores CCD e HRC.

Quadro 1: Características das imagens orbitais dos sensores CCD e HRC.

\begin{tabular}{|c|c|c|c|}
\hline Satélite/Sensor & $\begin{array}{c}\text { Resolução } \\
\text { Espacial }\end{array}$ & $\begin{array}{c}\text { Faixa } \\
\text { Imageada }\end{array}$ \\
\hline
\end{tabular}




\begin{tabular}{|l|l|l|c|}
\hline & & $2-0,45-0,52 \mu \mathrm{m}$ (azul) & \multirow{2}{*}{$113 \mathrm{~km}$} \\
& $20 \mathrm{~m}$ & $\begin{array}{l}3-0,52-0,59 \mu \mathrm{m} \text { (verde) } \\
4-0,63-0,69 \mu \mathrm{m} \text { (vermelho) }\end{array}$ & \\
\hline CBERS/HRC & $2,5 \mathrm{~m}$ & $1-0,50-0,80 \mu \mathrm{m}$ (pan) & $27 \mathrm{~km}$ \\
\hline
\end{tabular}

Fonte: INPE, 2015

Os procedimentos operacionais utilizados basearam-se na aquisição, armazenamento, manipulação, análise e apresentação de dados georreferenciados. A primeira etapa constituiu-se na criação de um Banco de Dados e Projeto identificando projeção cartográfica e delimitação do retângulo envolvente. Posteriormente foram aplicados alguns procedimentos de processamento nas imagens dos sensores CCD e HRC como: registro, recorte de plano de informação, restauração, reamostragem (Figura 2).

Figura 2: Características das imagens orbitais dos sensores CCD e HRC.

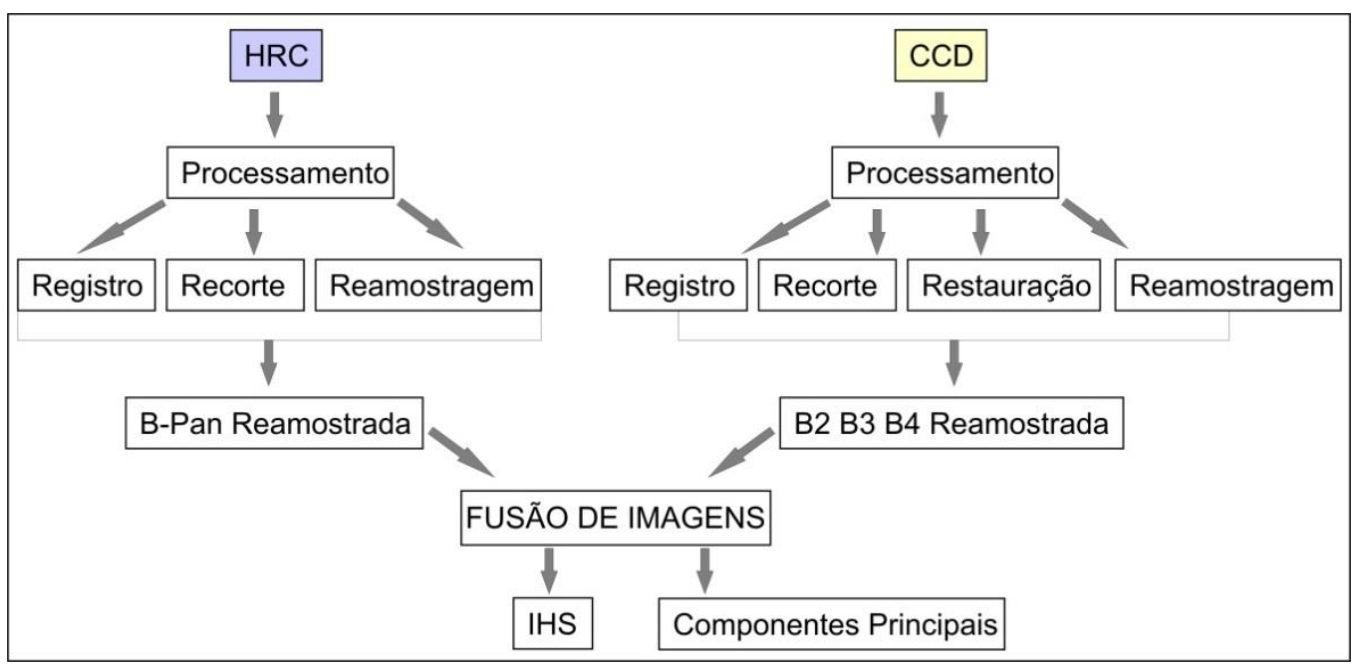

Organização: Cesar Cardoso Ferreira

A restauração corrige as distorções inseridas pelo sensor na geração das imagens e tem parâmetros de acordo com as características do sensor. O uso desta técnica permite gerar a partir da imagem de uma banda CCD com resolução espacial de 20 metros uma nova imagem com resolução espacial de 10 metros. No processo de reamostragem da imagem do sensor CCD adquiriu o mesmo tamanho de pixel da cena HRC, fazendo uso do interpolador bilinear (IBAMA/SISCOM).

Em seguida foram aplicadas as técnicas de IHS e Componentes Principais. Na transformação RGB para IHS, as cores são obtidas de uma composição colorida 
de bandas CCD, e na execução da transformação inversa IHS para RGB, a componente I (intensidade) é substituída pela imagem HRC.

De acordo com Araújo (2009), a transformação IHS é uma técnica de processamento de imagens para a reamostragem dos pixels. Através dessa transformação é possível fusionar imagens de resoluções espaciais distintas proporcionando maior descrição dos elementos das imagens, no que se refere aos pixels.

A técnica de fusão de imagens por componente principal baseia-se na substituição da primeira componente pela imagem de resolução maior, a qual pode ser contrastada de forma a ter média e variância próximas às da primeira componente.

\section{RESULTADOS}

A partir da aplicação das técnicas IHS e Componentes principais foram gerados duas imagens sintéticas fusionadas. Imediatamente notou-se um refinamento na escala dos objetos encontrados na superfície de estudo processadas pela técnica de fusão das imagens do sensor CCD com o HRC condicionando melhor possibilidade de interpretação visual das imagens.

A imagem sintética gerada pela técnica IHS apresentou nitidez e contraste realçados diferenciando os objetos encontrados na área de estudo. Porém em alguns alvos notaram-se diferenças largas e acentuadas nas transições dos pixels (Figura 3).

Figura 3: Comparação das imagens geradas pelos procedimentos IHS e Componentes Principais. 


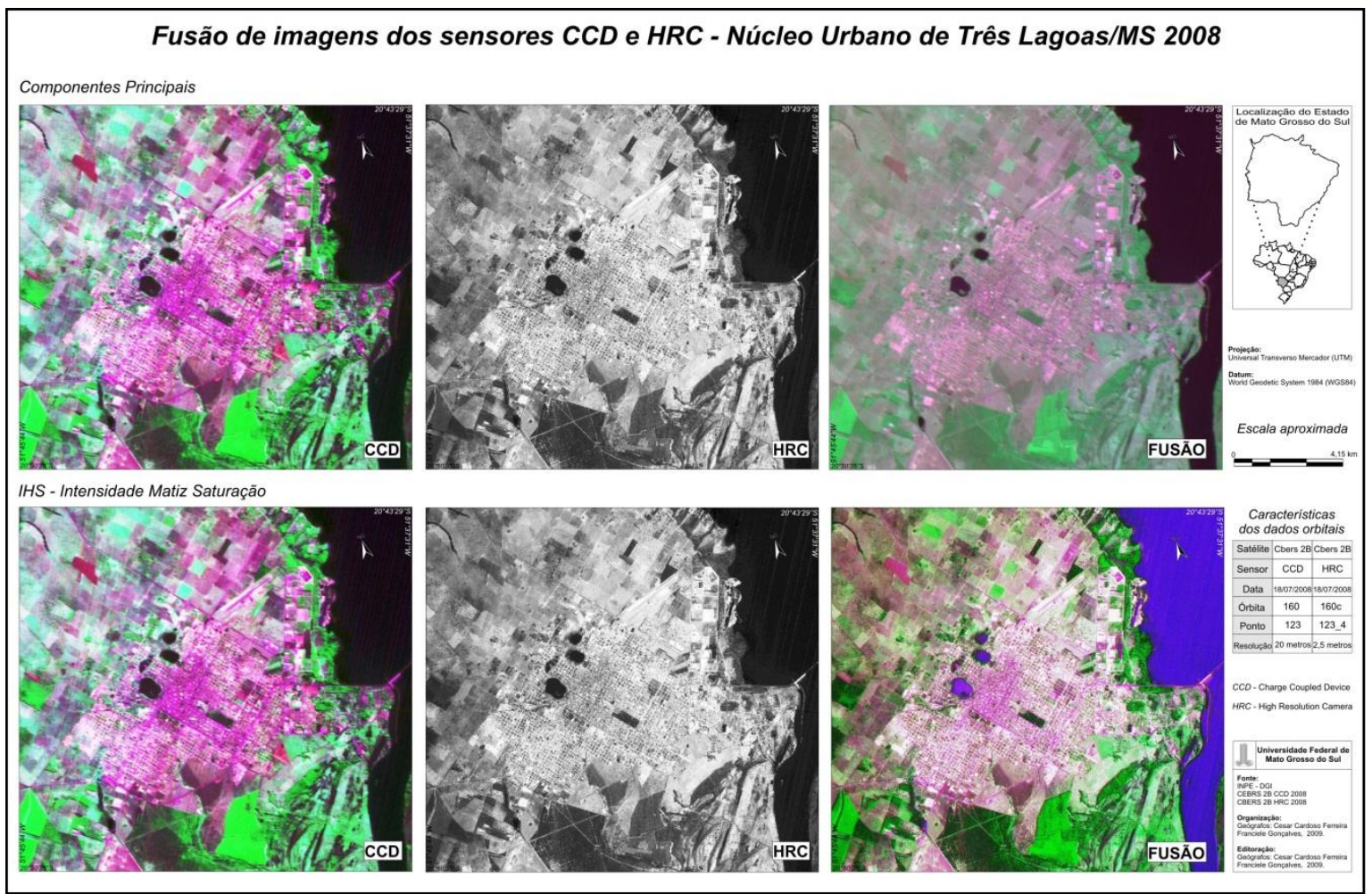

Fonte: Imagens CEBRS CCD e HRC, 2008. Organização: Cesar Cardoso Ferreira

A imagem sintética gerada pela técnica de componentes principais apresentou um realce que reduziu a difusão espectral, ou seja, gerou um novo conjunto de imagens cujas bandas individuais apresentaram informações nãodisponíveis em outras bandas.

As imagens de componentes principais podem ser combinadas em cores, como quaisquer outras. Quando comparada com qualquer combinação das bandas originais, a composição colorida (RGB) das componentes principais apresenta um realce na distribuição das cores, uma vez que não há correlação entre as bandas.

Os histogramas de contraste das imagens sintéticas geradas a partir da fusão pelos procedimentos IHS e Componentes Principais foram equalizados obtendo uma melhor qualidade visual das imagens (Figura 4).

Figura 4: Comparativo de imagens fusionadas e não fusionadas 


\section{Comparativo do comportamento de alguns alvos orbitais fusionados e não fusionados}

A

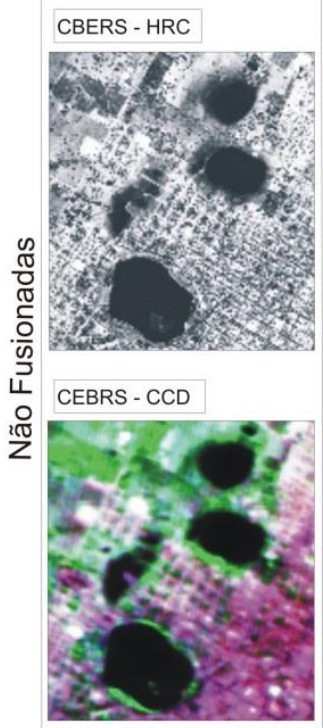

B
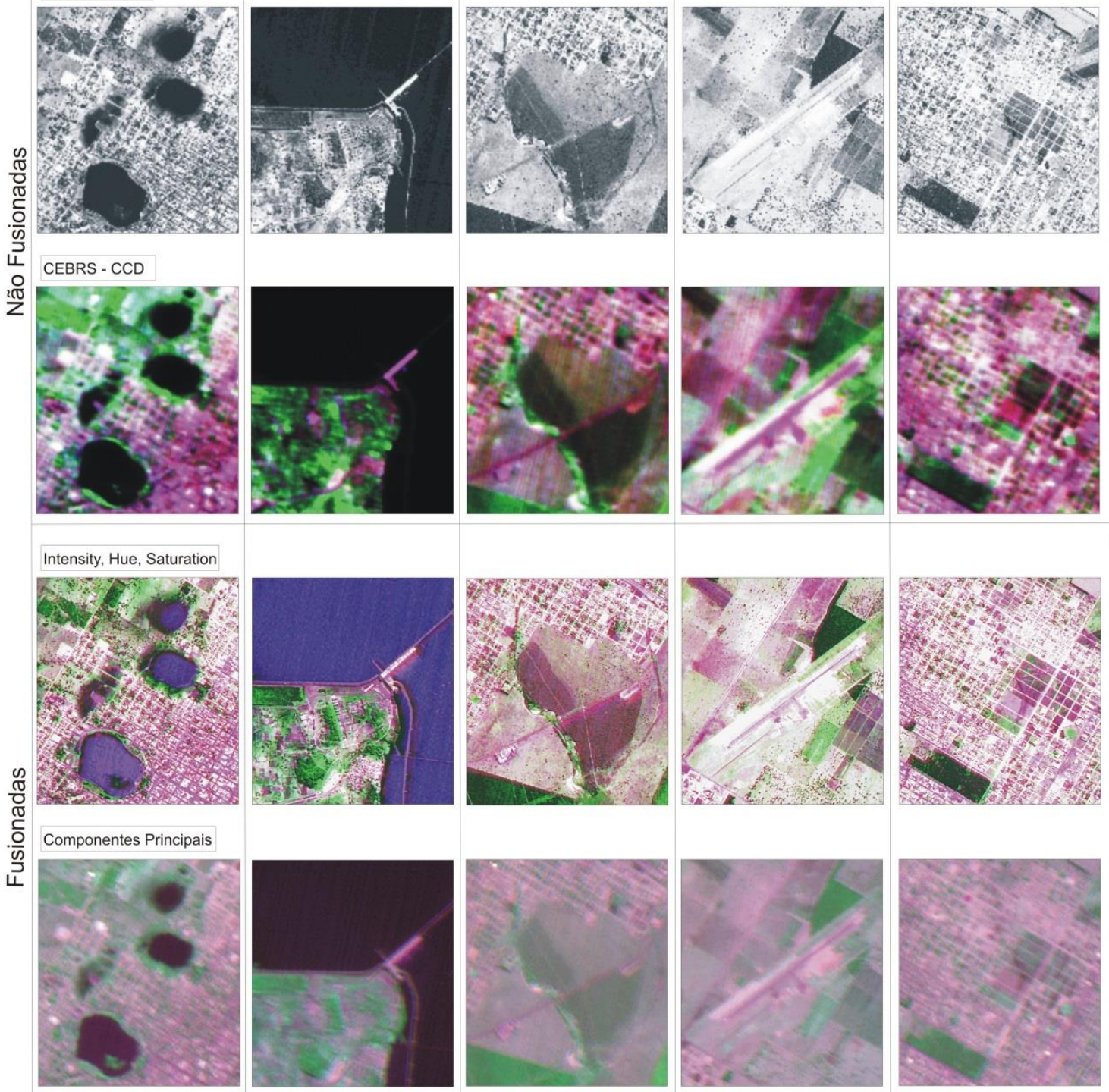

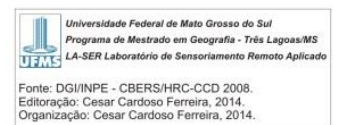

Organização: Cesar Cardoso Ferreira

A figura 4 evidencia que as imagens geradas pelos diferentes procedimentos são mais nítidas se comparadas com as imagens do sensor CCD e HRC. Essa nitidez proporciona facilidade na identificação e interpretação dos alvos encontrados na superfície de estudo, tornando possível a elaboração de mapeamentos temáticos com maiores precisões. 


\section{CONCLUSÕES}

Pelas informações apresentadas na área urbana de Três Lagoas, a imagem sintética gerada pela técnica Intensity Hue Saturation (IHS) apresentou melhor nitidez comparada à imagem sintética gerada pelo modo de Componentes Principais.

Em relação ao método utilizado ficou evidente sua eficácia, pois, a aquisição, manipulação e armazenamentos dos dados da área de estudo foram processados e transformados em informações com melhor resolução espacial.

Por fim, conclui-se que ambas as técnicas de fusão de imagem apresentaram resultados satisfatórios para identificação e distinção de alvos na área urbana de Três Lagoas-MS proporcionando melhores produtos orbitais para análises diversas.

\section{REFERÊNCIAS BIBLIOGRÁFICAS}

ARAÚJO, de C. M. H. M et al. Aplicação da transformação IHS para reamostragem de pixels, utilizando as imagens dos sensores CCD e HRC do satélite CBERS. Anais XIV SBSR. Natal, 2009.

ASSAD, E. D.; SANO, E. E. Sistemas de Informação Geográficas: Aplicações na Agricultura. Platina: Embrapa, 1993

BONHAN-CARTER, Graeme. Geographic information systems for geoscientists: modelling with GIS. Canadá. 1994

CÂMARA, G.; MEDEIROS, C.B.; CASANOVA, M.A.; HERMELY,A.; MAGALHÃES, G. Anatomia de Sistemas de Informação Geográfica. Campinas, Instituto de Computação/UNICAMP. (1996)

FEREIRA, Cesar Cardoso; ÇONÇALVES, Franciele, SAKAMOTO, Arnaldo Yoso; MIRANDOLAAVELINO, Patrícia Helena. Urban Area Analysis at Drainage Basin of Pitangueiras Stream, in Barretos City, São Paulo State, Brazil, Using Actives (SRTM) and Passives (LANDSAT ETM+) Orbitals Sensing. In: ELEVENTH URSI COMMISSION F TRIENNIAL OPEN SYMPOSIUM ON RADIO WAVE PROPAGATION AND REMOTE SENSIN, 2007, Rio de Janeiro-RJ. Anais

FERREIRA, C. C. GEOPROCESSAMENTO E SENSORIAMENTO REMOTO: Processamento de Imagens Orbitais de Sensores Passivos (CCD e TM) e Ativos (SRTM) como Subsídio para o Gerenciamento da Bacia Hidrográfica das Pitangueiras/SP. Monografia do Curso de Bacharelado em Geografia - UFMS, campus de Três Lagoas, 2008.

FERREIRA, C. C. Geotecnologias aplicada a criação e organização de banco de dados geoambientais da bacia hidrográfica do Rio Sucuriú - MS/BR. Mestrado em Geografia Universidade Federal de Mato Grosso do Sul, Três Lagoas, 2011.

GIRARD, Michel Claude; GIRARD Colette M. Traitement des donnéss de télédétection. Paris. 1999. 
INPE - Instituto Nacional de Pesquisas Espaciais. (2000) "Fundamentos de Geoprocessamento". Tutorial - SPRING.

INPE - Instituto Nacional de Pesquisas Espaciais. DPI acesso em 18/03/2015 disponível em http://www.dpi.inpe.br

NOVAES, Maikon R. de. Et all. Técnica de Fusão de Imagens para Facilitar a Detecção de Áreas Canavieiras em Incompatibilidade Ambiental. In: GEOINFO - X Brazillian Symposium on Geoinformatics, Rio de Janeiro, 2008.

NOVO, E. M. L. M. Sensoriamento Remoto: princípios e aplicações. São Paulo: Edgard Blucher, 2008.

WALD, L. Some terms of reference in data fusion. IEEE Transaction on Geoscience and Remote Sensing. v. 37, n. 3, p.1190-1193, 1999.

ROSA, Roberto. BRITO, José Luis Silva. Introdução ao geoprocessamento: Sistema de Informação Geográfica. Uberlândia, 1996.

V 